\title{
Sleep pattern and learning in knockdown mice with reduced cholinergic neurotransmission
}

\author{
C.M. Queiroz ${ }^{1,2,3 *}$, P.A. Tiba ${ }^{2,4 *}$, K.M. Moreira ${ }^{2}$, P.A.M. Guidine ${ }^{5}$, G.H.S. Rezende ${ }^{5}$, \\ M.F.D. Moraes ${ }^{5}$, M.A.M. Prado ${ }^{6}$, V.F. Prado ${ }^{6}$, S. Tufik ${ }^{2}$ and L.E. Mello ${ }^{1}$ \\ ${ }^{1}$ Departamento de Fisiologia, Universidade Federal de São Paulo, São Paulo, SP, Brasil \\ ${ }^{2}$ Departamento de Psicobiologia, Universidade Federal de São Paulo, São Paulo, SP, Brasil \\ ${ }^{3}$ Instituto do Cérebro, Universidade Federal do Rio Grande do Norte, Natal, RN, Brasil \\ ${ }^{4}$ Centro de Matemática, Computação e Cognição, Universidade Federal do ABC, Santo Andre, SP, Brasil \\ ${ }^{5}$ Núcleo de Neurociências, Departamento de Fisiologia e Biofísica, Universidade Federal de Minas Gerais, \\ Belo Horizonte, MG, Brasil \\ ${ }^{6}$ Molecular Brain Research Group, Robarts Research Institute, Department of Physiology and Pharmacology and Department of \\ Anatomy and Cell Biology, University of Western Ontario, London, Ontario, Canada
}

\begin{abstract}
Impaired cholinergic neurotransmission can affect memory formation and influence sleep-wake cycles (SWC). In the present study, we describe the SWC in mice with a deficient vesicular acetylcholine transporter (VAChT) system, previously characterized as presenting reduced acetylcholine release and cognitive and behavioral dysfunctions. Continuous, chronic ECoG and EMG recordings were used to evaluate the SWC pattern during light and dark phases in VAChT knockdown heterozygous (VAChT-KD ${ }^{\mathrm{HET}}, \mathrm{n}=7$ ) and wild-type $(\mathrm{WT}, \mathrm{n}=7)$ mice. SWC were evaluated for sleep efficiency, total amount and mean duration of slow-wave, intermediate and paradoxical sleep, as well as the number of awakenings from sleep. After recording SWC, contextual fear-conditioning tests were used as an acetylcholine-dependent learning paradigm. The results showed that sleep efficiency in VAChT-KD ${ }^{\mathrm{HET}}$ animals was similar to that of WT mice, but that the SWC was more fragmented. Fragmentation was characterized by an increase in the number of awakenings, mainly during intermediate sleep. VAChT$\mathrm{KD}^{\mathrm{HET}}$ animals performed poorly in the contextual fear-conditioning paradigm (mean freezing time: $34.4 \pm 3.1$ and $44.5 \pm 3.3 \mathrm{~s}$ for WT and VAChT-KD ${ }^{\mathrm{HET}}$ animals, respectively), which was followed by a $45 \%$ reduction in the number of paradoxical sleep episodes after the training session. Taken together, the results show that reduced cholinergic transmission led to sleep fragmentation and learning impairment. We discuss the results on the basis of cholinergic plasticity and its relevance to sleep homeostasis. We suggest that VAChT-KD ${ }^{\mathrm{HET}}$ mice could be a useful model to test cholinergic drugs used to treat sleep dysfunction in neurodegenerative disorders.
\end{abstract}

Key words: Sleep-wake cycle; Intermediate sleep; Acetylcholine; Contextual fear conditioning; Memory; Neurodegenerative disorders

\section{Introduction}

Cholinergic neurotransmission plays a key role in many physiological processes in the central nervous system, ranging from learning and memory formation to fine control of sleep-wake cycles (SWC). Cholinergic neurons in the basal forebrain and brain stem pedunculopontine tegmentum (PPT) areas project and diffusely innervate the entire neocortex, amygdala, hippocampus, thalamus, and the midbrain (1). Exogenous excitation of
PPT cholinergic cells can increase wakefulness and/or paradoxical sleep (PS) in a dose-dependent manner (2), while episodes of spontaneous waking and PS are accompanied by increased activity of cholinergic neurons $(3,4)$. During waking, when animals are behaviorally active, cholinergic activation produces fast electroencephalographic activity in the cortex (5) that has been associated with periods of alertness, exploration and

Correspondence: L.E. Mello, Departamento de Fisiologia, UNIFESP, Rua Pedro de Toledo, 669, 3ํandar, E.C.B., 04039-032 São Paulo, SP, Brasil. E-mail: lemello@unifesp.br

*These authors contributed equally to this study. 
learning (6). On the other hand, cholinergic activity reaches a minimum during slow-wave sleep (SWS) (4). Taken together, these observations suggest that cholinergic activation during waking and PS can modulate cortical activity favoring plasticity and synaptic reorganization (7).

The idea that not only wakefulness, but also sleep, plays a role in plasticity, synaptic reorganization and memory consolidation is extensive; however, the mechanisms involved are still a matter of intensive debate. On the one hand, sleep patterns are modified by learning paradigms, and those changes are believed to enhance memory consolidation (8). On the other hand, sleep deprivation impairs several cognitive processes, such as memory function, motor performance, mood, and executive attention (9). Acetylcholine (ACh) antagonists, such as scopolamine, impair learning and memory (1), while high levels of ACh during wakefulness are necessary to encode long-term memories (10). In fact, it has been shown that fluctuations of ACh concentration during sleep stages (i.e., low during SWS and high during REM sleep) in humans are fundamental for proper memory consolidation (11). Interestingly in rats, sleep deprivation-induced memory impairment can be reversed by administration of the cholinergic agonist pilocarpine (12).

In humans, cholinergic dysfunction is highly associated with disrupted sleep (13) and impaired memory formation $(10,14)$. Elderly patients are among the most affected population, in whom severe dementia, including Alzheimer's type, reduces the number of cholinergic neurons (15), possibly causing various types of sleepwake disorders, including irregular SWC (16), difficulty in falling or staying asleep, or adhering to a consistent sleep/wake schedule (17). Since suboptimal sleep greatly contributes to the deterioration of health and reduction in the quality of life of neurological patients, there is a need for a better understanding of the contribution of cholinergic tone to mechanisms involved in sleep control (18).

In the last decade, different transgenic mice were produced and used to further evaluate the contribution of cholinergic neurotransmission in sleep generation, maintenance and control. For example, mice lacking the beta2 subunit gene of the nicotinic ACh receptor are insensitive to nicotine-induced alertness, and have impaired PS (19), while mice with a hypersensitive alpha-4 nicotinic receptor subunit have altered SWC and are seizure prone (20). Finally, genetically modified mice $>6$ months of age expressing high levels of betaamyloid peptides (Tg2576 mice) show impaired PS and a significant reduction in the number of choline acetyltransferase-positive neurons in the PPT and cognitive impairments (21).

Given the diversity of muscarinic and nicotinic receptors, it is likely that some of the effects of cholinergic deficiency cannot be mimicked in mouse knockouts of individual receptor subunits. Here we evaluate SWC and the behavioral performance in the contextual fearconditioning (CFC) test in animals with reduced cholinergic tone. Hypocholinergia was achieved by reducing the expression of the vesicular ACh transporter (VAChT), a key protein that regulates synaptic vesicle release of ACh (22) and thus resembles specific aspects of cholinergicdependent senile dementia in humans. It was previously shown that heterozygous knockdown animals for VAChT present a $45 \%$ reduction in VAChT protein expression, which results in at least a $30 \%$ reduction in extracellular concentration of ACh in the frontal cortex and striatum (22). Our working hypothesis was that reduced cholinergic tone would influence both SWC and memory processes and that sleep alterations taking place after learning could be further used to strengthen the sleep-memory relationship.

\section{Subjects and Methods}

\section{Subjects}

Adult (4 to 6 months old), male, aged-matched wildtype (WT) and knockdown heterozygous ACh transporterdeficient (VAChT-KD ${ }^{\mathrm{HET}}$ ) (22) mice were used in the present experiments $(n=7 /$ group, unless otherwise stated). Generation and genotyping of the mice have been described elsewhere $(22,23)$. In summary, these mice were generated by targeting the $5^{\prime}$-untranslated region of the VAChT gene by homologous recombination in a mixed 129S6/SvEvTac (129S6) × C57BL/6J background, and were backcrossed to $\mathrm{C} 57 \mathrm{BL} / 6 \mathrm{Uni}$ mice (imported from Zentralinstitut fuer Versuchstierzucht, Hannover, Germany) for three generations (N3), as further backcrossing caused infertility. Therefore, a mixed background was maintained in the population by preferentially mating animals that were not closely related. Assessment of cholinergic function in these mice by in vivo microdialysis and electrophysiological recording at the neuromuscular junction indicated a $40-50 \%$ decrease in the release of $\mathrm{ACh}$ (22). The VAChT protein is required for ACh transport in synaptic vesicles and for its release by exocytosis (24). Animals were housed in groups of 5 to 6 animals in plexiglass cages with free access to rat food pellets and tap water in a room with controlled temperature $\left(22^{\circ} \mathrm{C}\right)$ and with a $12: 12$-h light-dark cycle with lights on at 7:00 am. The experimental procedures used in the present experiments were in accordance with the Guidelines for the Care and Use of Animals of the US National Institutes of Health and the Animal Care and Use Ethics Committee of the Universidade Federal de São Paulo (UNIFESP). Efforts were made to reduce the number of animals used in the present experiments without compromising the statistical analyses.

\section{Surgery}

Mice were anesthetized with a mixture of ketamine/ xylazine $(100 / 10 \mathrm{mg} / \mathrm{kg}$, ip) and after checking for the 
absence of hindpaw pain reflex, they were placed in a stereotaxic frame. After exposing the skull, four stainless steel screws were placed above the dura mater of the parietal cortex (above the hippocampus) in both hemispheres for electrocorticographic (ECoG) recordings. The following coordinates were used for each screw relative to the bregma (antero-posterior and medio-lateral coordinates, respectively): screw 1: $0.5,0.8 \mathrm{~mm}$; screw $2:-1.2$, $1.8 \mathrm{~mm}$; screw 3: $-0.5,-0.5 \mathrm{~mm}$; screw 4: $-2.0,-2.0 \mathrm{~mm}$. Bipolar derivations (screws 1 vs 2, and screws 3 vs 4; ipsilateral derivations) were used to access and classify brain activity in both hemispheres. Electromyographic (EMG) recordings from the dorsal neck muscles of the mice allowed verification of muscular tonus and aided in the classification of various times within the SWC. At the end of the surgical procedure, all mice received an injection of a broad-spectrum antibiotic $(0.5 \mathrm{~mL} / \mathrm{kg}$ Pentabiótico, sc, Fort Dodge, Brazil) to minimize postsurgical infection. Animals were allowed to recover from surgery in their home cages for at least 15 days before any experimental procedures were performed.

\section{Sleep-wake cycle recording}

Animals were placed individually in the recording chamber and connected to an ECoG polygraph using a light-weight cable 5 days before the recordings started. After the habituation period, ECoG and EMG recordings to determine SWC characteristics were started. Signals were differentially amplified and sampled at 100 or $200 \mathrm{~Hz}$. ECoG was filtered between 0.1 and $60 \mathrm{~Hz}$; EMG was filtered between 0.1 and $100 \mathrm{~Hz}$. The signals were displayed on a computer screen and stored on a hard disk for offline analysis using the Somnologica ${ }^{\mathbb{R}}$ software (Medcare, Iceland). Animals were continuously recorded for at least 3 days, and up to 5 days, for the quantification of baseline sleep-wake parameters.

\section{Data analysis}

ECoG and EMG traces were used to visually score the different behavioral states of the SWC, i.e., awake (W), SWS, intermediate sleep (IS), and paradoxical sleep (PS). The traces were classified on the basis of the dominant $(>50 \%)$ pattern within a 30 -s scoring window (epoch). Visual inspection of the recordings was assisted by plotting the frequency power spectrum (fast Fourier transform, 1024 points) of the middle third (10 s) epoch of the scoring window. Auto- and cross-correlations between ECoG recordings determined with Matlab (MathWorks, USA) were used to further characterize the different brain states and to calculate the relative power of specific frequency bands over extended time periods (>24 h). Waking epochs were characterized by desynchronized ECoG, absence of similarity between waveforms from both hemispheres, and increased muscular tonus (EMG). SWS was characterized by high delta $(0.5-4 \mathrm{~Hz})$ power in the ECoG combined with reduced EMG amplitude and mild cross-correlation between recordings from the two hemispheres. PS was scored when ECoG presented oscillations in the theta range (4-9 Hz) and EMG indicated reduced muscular tonus. We also included an additional stage, IS, which was characterized by the presence of slow wave oscillations, sleep spindles $(10-14 \mathrm{~Hz})$ and two peaks of theta activity, at 4 and $7 \mathrm{~Hz}$ with comparable power (25).

Classification of brain states allowed the computation of different parameters of the SWC of WT and VAChT$\mathrm{KD}^{\mathrm{HET}}$ mutant animals. For each animal, data from different days were averaged into one single period of $24 \mathrm{~h}$. In the comparisons made, we considered the sleep efficiency (percentage of total sleep time during the recording time), the percentage of total sleep time, the number of episodes, and the mean duration of each sleep state (SWS, IS, and PS). In order to evaluate the stability and maintenance of sleep behavior, we quantified the number of awakenings from each sleep state (SWS, IS and PS). We distinguished between two types of awakenings, those which were brief in time $(<60 \mathrm{~s})$ in which no gross movements were observed aside from small adjustments of body position and those in which animals engaged in any other behavior than sleep, i.e., ambulation, eating, drinking, and/or grooming. Data from light and dark phases of the day were analyzed separately (each one consisting of a 12-h period) when appropriate.

Previous studies have established that a reduction of up to $50 \%$ in VAChT levels does not affect neuromuscular function $(22,23)$. The reason for that is the high safety margin for neuromuscular transmission. We studied VAChT-KD ${ }^{\text {HET }}$ mice that had a $40 \%$ reduction in VAChT levels. Therefore, those mice were as physically fit as WT mice (22).

\section{Behavioral paradigm}

After the basal recordings of the SWC, learning and memory were evaluated using the CFC test. This behavioral paradigm has been associated with increased release of ACh in the hippocampal region (26). The CFC test apparatus consisted of an acrylic box measuring $30 \times 21 \times 30 \mathrm{~cm}$. The apparatus had black walls with white visual patterns (2 squares measuring $5.5 \times 5.5 \mathrm{~cm}$ and 3 measuring $4.0 \times 4.0 \mathrm{~cm}$ and made of white cardboard). The top was covered with transparent acrylic. The floor consisted of a metal grid $(0.4-\mathrm{cm}$ diameter rods placed $1.2 \mathrm{~cm}$ apart) connected to a shock generator and control module (AVS, Projetos Especiais, Brazil), by which foot shocks could be delivered. On the day of the experiment, animals were disconnected from the ECoG recording and dislocated to one contiguous room for CFC training. Animals were individually placed in the conditioning apparatus, where they remained undisturbed for $2 \mathrm{~min}$. Two minutes later, 3 foot shocks $(0.8 \mathrm{~mA}, 1 \mathrm{~s})$ were delivered with a 30 -s interval between each shock. Two minutes after the last foot shock, the animal was removed 
from the apparatus, returned to the recording cage, and reconnected to the ECoG setup. It has been shown that performance in the CFC test is sensitive to sleep deprivation (27). Thus, animals were recorded until the next day to evaluate shock-induced behavioral and sleep alterations. We hypothesized that reduced $\mathrm{ACh}$ release in VAChT-KD ${ }^{\mathrm{HET}}$ animals would contribute to impaired test performance on the following day. The test was performed on the second day, $24 \mathrm{~h}$ after the training. Each animal was returned to the conditioning apparatus, but no foot shock was applied. An experienced investigator recorded freezing time - defined as complete immobility of the animal, with the absence of vibrissae movements and sniffing - in minute bins continuously for 5 min with a chronometer. Both training and testing occurred in the afternoon, starting at 1:00 pm and lasted up to $2 \mathrm{~h}$.

\section{Statistical analysis}

Data were normalized over 12- or 24-h periods and reported as means \pm SE. Comparisons between WT and mutant mice were made using two-way ANOVA (when applicable) and/or the Student $t$-test as post hoc comparisons. Analysis of correlation was used to check whether behavioral performance in the testing session correlated with the sleep profile following the training session.

\section{Results}

\section{Sleep-wake classification}

ECoG and EMG profiles used in the classification of the SWC are shown in Figure 1. In the 30-s resolution window, behavioral state was scored according to standard criteria. During W states, ECoG showed desynchronized activity and high EMG tonus. Occasionally, oscillations in the theta frequency were recorded. SWS was characterized by ECoG slow activity (maximum power $\sim 2 \mathrm{~Hz}$ ) and decreased muscle tone. During IS, ECoG recorded delta waves and spindles mixed with theta activity. Power analysis of this rhythm showed two peaks with similar energy, one at $3-4 \mathrm{~Hz}$ and the other at $6-8 \mathrm{~Hz}$. Interestingly, at this stage, the synchronization between hemispheres was greatly reduced (Figure $1 \mathrm{C}$, cross-correlation, third line) and this information was further used in SWC classification. Finally, PS was characterized by both synchronous theta activity in the ECoG and muscle atonia. Theta activity during this state peaked between 6 and $7 \mathrm{~Hz}$. The time course of the three main frequency bands (delta, theta, and sigma) in relation to SWC classification is shown in Figure 2A. Relative delta, theta and sigma power and absolute EMG power were averaged for each animal in each of the SWC states ( $n=4 /$ group). Two-way ANOVA revealed no effect of condition (WT vs VAChT-KD ${ }^{\mathrm{HET}}$ : $\left.F_{(1,24)}<1.39 ; P>0.05\right)$ for all frequency bands and thus, data from all animals were grouped. On the other hand, grand averages of relative delta, theta and sigma power varied accordingly to the SWC. As expected, relative delta $\left(F_{(3,24)}=44.24 ; P<0.001\right)$, theta $\left(F_{(3,24)}=30.14\right.$; $\mathrm{P}<0.001)$ and spindles $\left(\mathrm{F}_{(3,24)}=50.90 ; \mathrm{P}<0.001\right)$ power were significantly higher in SWS, PS and IS, respectively (Figure 2B). Combined with absolute EMG power (higher during wake), these frequency bands allowed satisfactory and reliable sleep classification and thus, they were used in the following analysis of the SWC of VAChT-KD ${ }^{\mathrm{HET}}$ animals.

\section{Sleep-wake cycle}

Quantification of SWC during 3-5 days showed that the sleep profile was not notably different in VAChT$\mathrm{KD}^{\mathrm{HET}}$ compared to WT animals (Figure 3). Sleep efficiency, defined as the time spent sleeping/total time, was $50 \%$ higher during the light phase than in the dark phase (effect of phase: $F_{(1,24)}=32.95$; $P<0.0001$; twoway ANOVA; Figure $3 \mathrm{~A}$ ), but not statistically different between groups. The control and experimental groups did not differ in the percentage of time spent in SWS, IS or PS during the dark (Figure 3B) and light (Figure 3C) phases. As expected, the percentage of time spent in PS in the light phase was higher during the light than the dark phase for both groups (effect of phase: $F_{(1,24)}=17.59$; $P<0.0005$ and $\mathrm{F}_{(1,24)}=7.40 ; \mathrm{P}<0.05$; two-way ANOVA; for SWS and PS, respectively). The average duration of each of the behavioral states was not different between groups (Figure 3D and E). Wake duration was two times longer in the dark phase (Figure 3D) compared with the light phase (Figure 3E) in both groups (effect of phase: $F_{(1,24)}=14.79$; $\mathrm{P}<0.001$, two-way ANOVA). No alteration in the mean duration of SWS, IS or PS was observed between light or dark phases or between groups, although a slight tendency to decreased mean duration of the SWS was observed in VAChT-KD ${ }^{\mathrm{HET}}$ animals, which was more evident in the light phase (Figure 3E, effect of the genetic background: $\left.F_{(1,24)}=3.80 ; P=0.063\right)$.

\section{Sleep fragmentation in VAChT-KD ${ }^{\mathrm{HET}}$}

In order to evaluate the amount of fragmentation in the SWC of VAChT-KD ${ }^{\mathrm{HET}}$ mice, we first quantified the number of transitions between two classified states. The average number of state transitions per hour during the dark phase was $16.1 \pm 2.3$ and $18.5 \pm 1.3$ and $22.8 \pm 2.2$ and $27.4 \pm 1.5$ during the light phase for WT and VAChT$\mathrm{KD}^{\mathrm{HET}}$ animals, respectively. The number of state transitions was significantly higher during the light than the dark phase (effect of circadian phase: $F_{(1,24)}=17.17$; $\mathrm{P}<0.0005$; two-way ANOVA), and VAChT-KD ${ }^{\mathrm{HET}}$ animals had only a tendency to have more state transitions when compared to WT animals (effect of genetic background: $F_{(1,24)}=3.48 ; P=0.074 ;$ two-way ANOVA). Since the state transitions were higher during the light phase, when the sleep drive is higher, we verified whether the increase in state transitions was associated with increased 


\section{A}

Wake

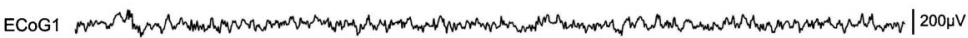

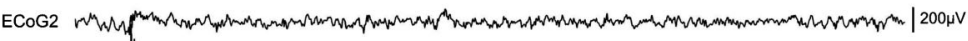

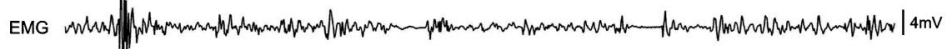

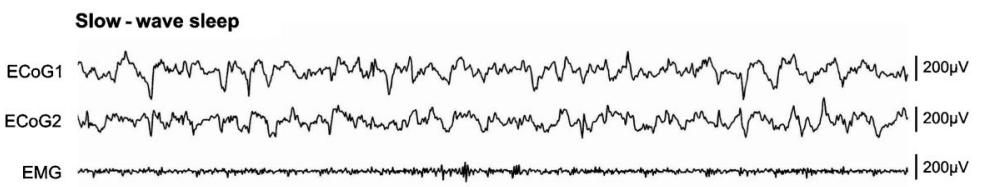

\section{Intermediate sleep}

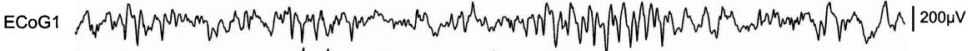

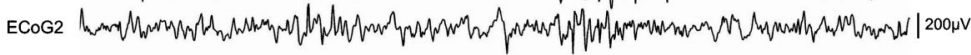
EMG

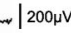

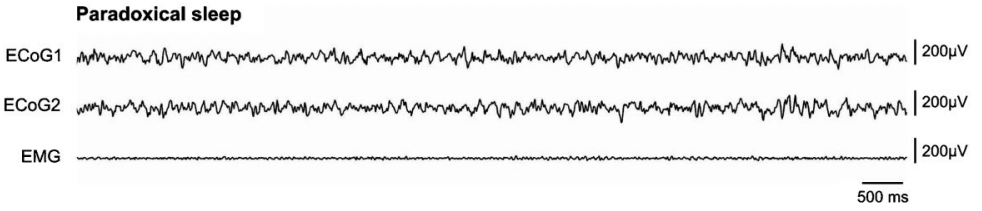

B
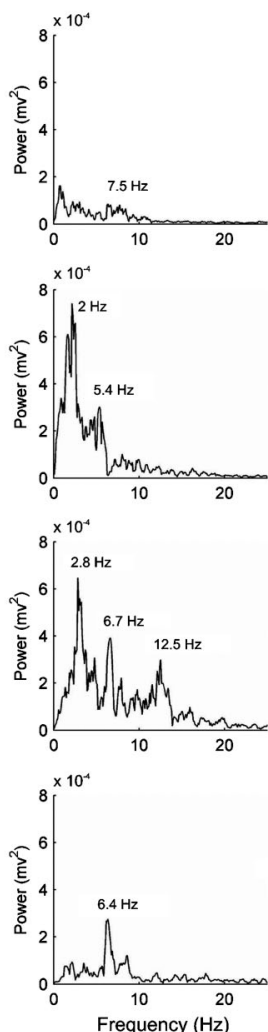
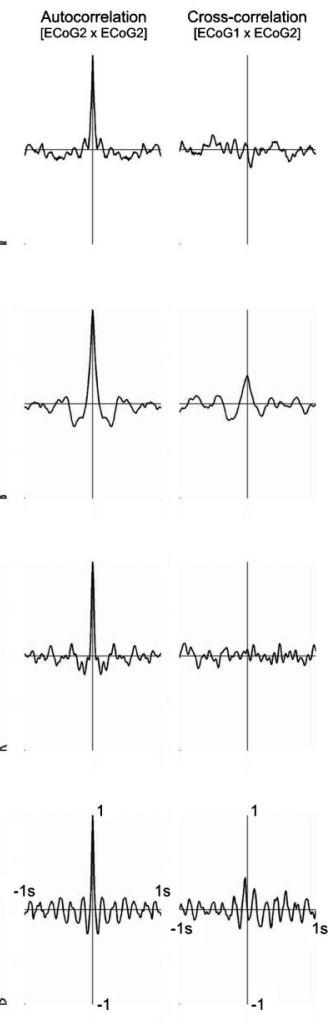

Figure 1. $A$, Representative traces of the four (wake, slow-wave sleep, intermediate sleep, and paradoxical sleep) identified electrocorticograms (ECoG1 and ECoG2) and electromyogram (EMG) patterns used to score the sleep-wake cycle. B, Fast Fourier transformation of the ECoG2 trace showing the peak frequencies in each state. $C$, Auto- (left column) and cross-correlations (right column) of the ECoG recordings.

awakenings.

Figure 4 shows the number of awakenings from different sleep stages during the dark and light phases. The number was higher during the light phase, when the amount of sleep was increased, than in the dark phase (effect of phase: $\mathrm{F}_{(1,24)}=8.20$; $\mathrm{P}<0.01$, two-way ANOVA). This assertion was also valid for longer (more than $60 \mathrm{~s}$ ) awakenings (effect of phase: $F_{(1,24)}=6.17$; $P<0.05$, twoway ANOVA). In fact, the number of awakenings that led to $\mathrm{W}$ periods longer than $60 \mathrm{~s}$ in the light phase was significantly increased in VAChT-KD ${ }^{\mathrm{HET}}$ animals (effect of genetic background: $F_{(1,24)}=5.896 ; P<0.05$, two-way ANOVA; Figure 4B, first column). Specifically, the number of IS periods followed by awakenings was significantly greater in VAChT-KD ${ }^{\mathrm{HET}}$ than in WT animals (effect of genetic background: $F_{(1,24)}=12.21$ and 10.90; $P<0.005$; for all, and for $>60 \mathrm{~s}$ awakenings, respectively) in both dark and light phases. The above results suggest that VAChT-KD ${ }^{\mathrm{HET}}$ animals showed an increased probability of awakening, thus fragmenting the sleep cycle, especially during IS.

\section{Behavioral paradigm: CFC and sleep-wake pattern}

The idea that sleep can assist memory consolidation has long been evaluated using different behavioral paradigms and electrophysiological analysis. In the present study, we analyzed the sleep alterations triggered by a brief session of CFC task training. Animals trained in this behavioral paradigm displayed an increased duration of freezing in the test session when evaluated $24 \mathrm{~h}$ after the training session. Notwithstanding, the mean freezing duration expressed as seconds per minute had a tendency to be decreased in VAChT-KD ${ }^{\mathrm{HET}}$ animals $(34.4 \pm 3.3$ vs WT: $44.5 \pm 3.1 ; \mathrm{P}=0.051$, Student $t$-test). This result indicates that animals with reduced ACh release present a mild impairment of memory formation in this behavioral paradigm.

In order to evaluate the hypothesis that memory impairment could be related, to some extent, to the impaired sleep profile after the training, we analyzed the sleep-wake pattern in the 4-h period after the training session (Figure 5 ). The training session produced alertness and consequent $\mathrm{W}$ behavior, for approximately 

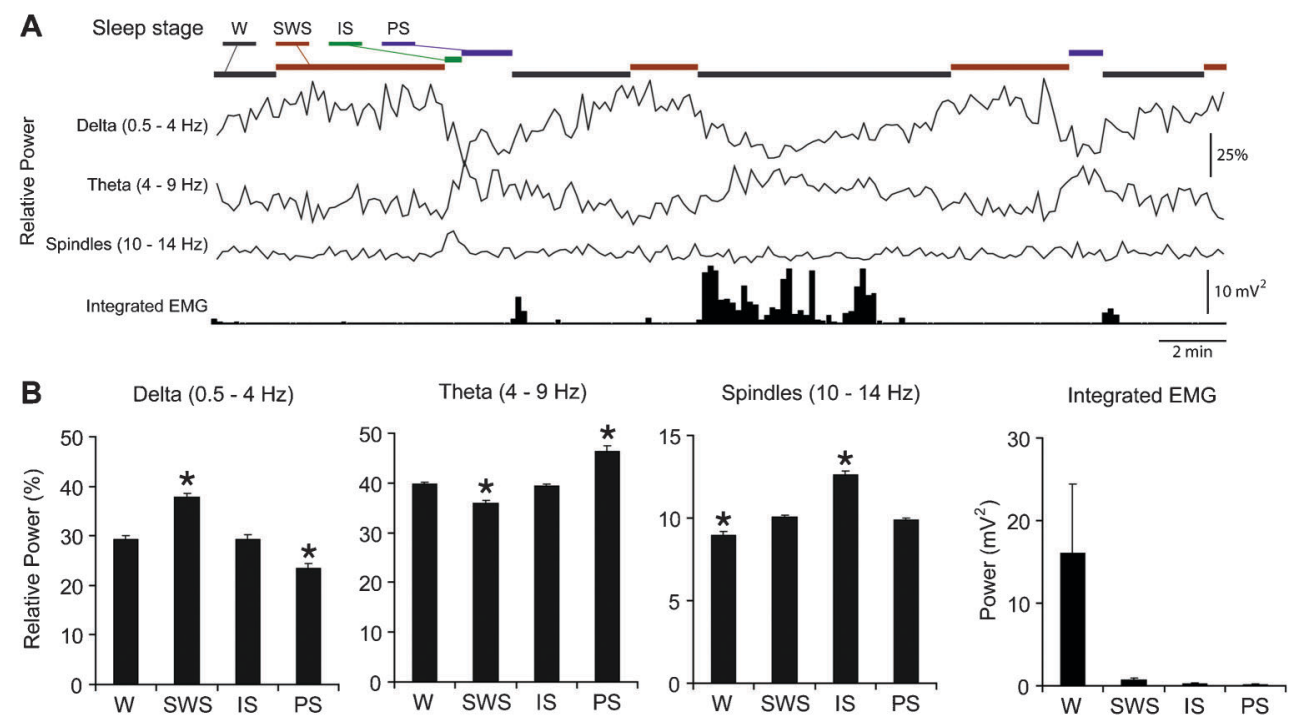

Figure 2. Time course of the sleep-wake cycles (SWC). A, Relative delta, theta and sigma power and absolute electromyogram (EMG) were computed using a 10-s sliding window fast Fourier transform and were correlated with scored hypnogram. This representative example shows the three most prevalent types of sleep transitions observed: slow-wave sleep (SWS) to intermediate sleep (IS) to paradoxical sleep (PS); SWS to PS and SWS alone. Note the increase in EMG power after an epoch of sleep. $B$, Grand averages of relative frequency bands across 8 mice $(n=4 /$ group) in each of the four SWC states recorded continuously during $48 \mathrm{~h}$ were calculated. As expected, delta oscillations were higher and lower during SWS and PS, respectively, while the opposite was observed during theta oscillations. Similarly, sleep spindles power was higher during IS and lower during awake (W). Data are reported as means \pm SE. ${ }^{*} \mathrm{P}<0.05$, compared to all other states (Bonferroni post hoc test).

20 min afterwards in both animal groups (Figure 5A). Sleep behavior started with SWS. After sleep initiation, the latency for IS and PS was not statistically different between groups (Figure 5A). However, the total duration of $W$ during the 4-h recording session was significantly longer in VAChT-KD ${ }^{\mathrm{HET}}$ animals (Figure 5D). Accordingly, the sleep efficiency was reduced by $25 \%$ in VAChT$\mathrm{KD}^{\mathrm{HET}}$ animals (Figure $5 \mathrm{C}$ ). This reduction was observed for all three different sleep stages, but the PS was the one with strongest reduction (reaching statistical significance; Figure 5D). Also, the number of PS episodes was significantly reduced by $45 \%$ in VAChT-KD ${ }^{\mathrm{HET}}$ animals after the training session (Figure $5 \mathrm{C}$ ). Interestingly, we observed a positive correlation between PS duration and freezing duration in the test session (Figure 5E). The correlation coefficient $(R)$ was higher for the cumulative freezing duration in the first 3 min of the test, which relates to the presentation of the shock during the training
A

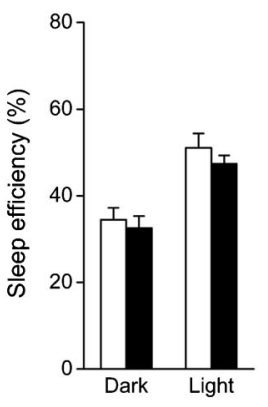

B

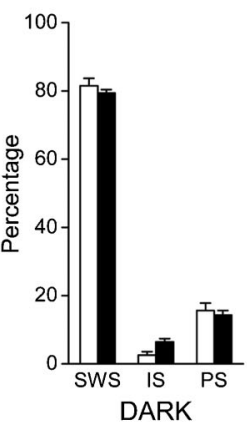

C

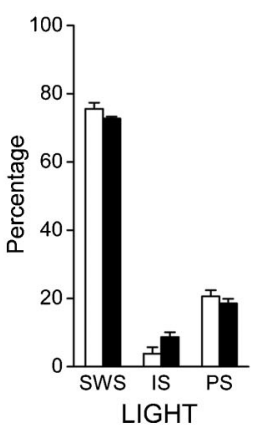

D

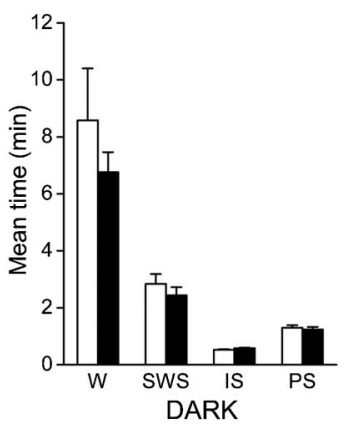

E

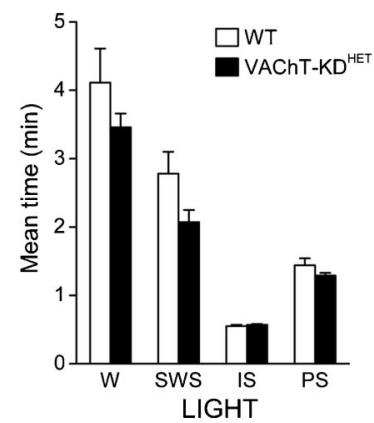

Figure 3. Circadian profile of the sleep-wake cycle. $A$, Sleep efficiency quantified during the dark and light phase (sleep efficiency \%: amount of time spent in sleep/total time). B,C, Relative amount of time spent in slow-wave sleep (SWS), intermediate sleep (IS) and paradoxical sleep (PS) during the dark $(B)$ and light $(C)$ phases. $D, E$. Averaged episode duration for each of the behavioral states quantified during the dark $(D)$ and light $(E)$ phases. WT: wild-type mice $(n=7)$; VAChT-KD ${ }^{\mathrm{HET}}$ : vesicular acetylcholine transporter knockdown heterozygous mice $(n=7)$; W: awake. Data are reported as means \pm SE. 
A

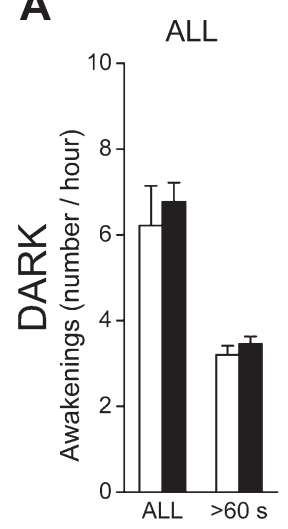

B

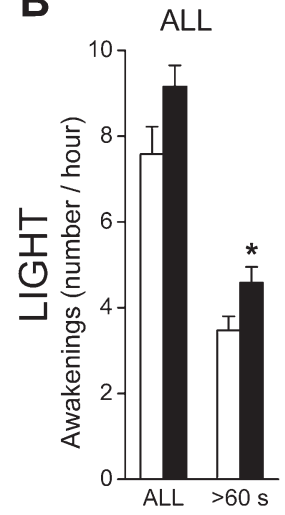

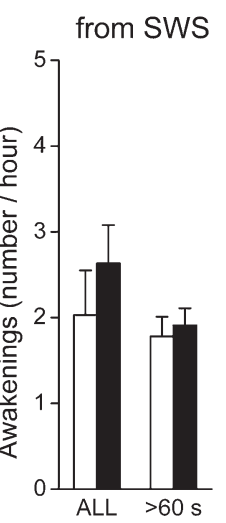

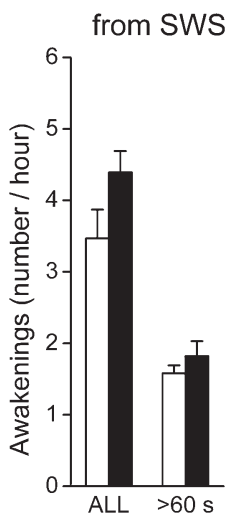

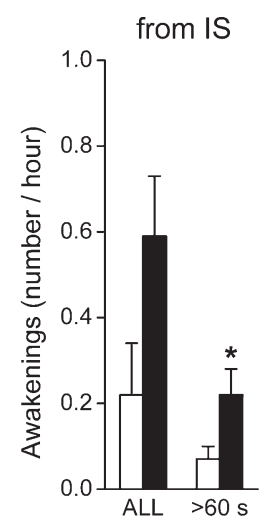

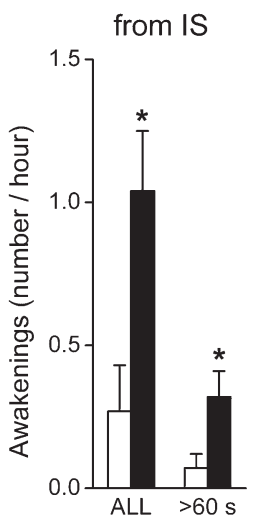

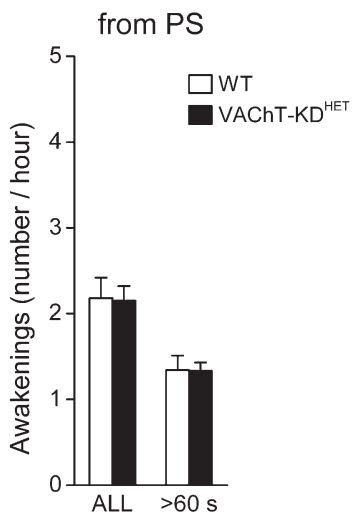

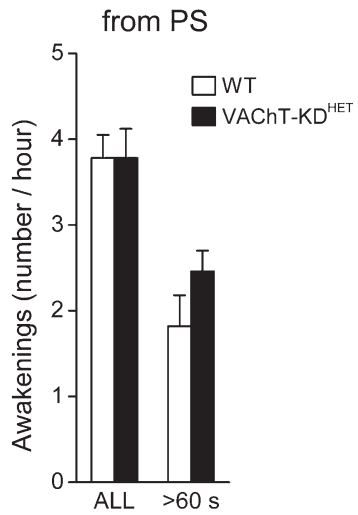

Figure 4. Awakenings during the dark $(A)$ and light $(B)$ phases. Data are reported as means \pm SE of the averaged number of awakenings in each 12-h period, separately from each sleep state. WT: wild-type mice $(n=7)$; VAChT-KD HET: vesicular acetylcholine transporter knockdown heterozygous mice $(n=7)$; SWS: slow-wave sleep; IS: intermediate sleep; PS: paradoxical sleep. ${ }^{*} P<0.05$, compared to WT (Student $t$-test).

session (between 2 and $3 \mathrm{~min}$ ). Taken together, these results suggest that behavioral manipulation (i.e., the training session and stress), possibly by challenging the cholinergic system, revealed an intrinsic characteristic of VAChT-KD ${ }^{\mathrm{HET}}$ animals for decreased global sleep duration, mainly affecting PS generation and/or maintenance.

\section{Discussion}

The present study showed that the baseline sleep efficiency of VAChT-KD ${ }^{\mathrm{HET}}$ animals did not differ from their WT counterparts. Nevertheless, the reduced cholinergic tone was responsible for increasing the number of awakenings during IS, thus preventing further development of PS. When exposed to mild stress, i.e., after being given foot shocks in a training session, VAChT-KD ${ }^{\mathrm{HET}}$ animals showed a reduced amount of sleep, mainly the PS stage. The possible relationship between performance in the behavioral test and sleep efficiency after CFC training is further discussed below.

\section{Sleep}

Reduced release of ACh, which characterizes VAChT$\mathrm{KD}^{\mathrm{HET}}$ animals (22), increased the number of awakenings, mainly during IS. These data are somewhat in accordance with the previous results obtained by Capece et al. (28) that showed that inhibition of the VAChT in the medial pontine reticular formation reduced the amount of PS. Even though our data showed no difference between groups at baseline, after a challenge (training), the amount of PS was reduced in VAChT-KD ${ }^{\mathrm{HET}}$ animals. The methodological differences between these studies (e.g., intact cats in the study by Capece et al. and knockdown mice in the present one) might be partly responsible for some of the differences in experimental outcome. Our results suggest that reduction of vesicular $\mathrm{ACh}$ transporter expression present in VAChT-KD ${ }^{\mathrm{HET}}$ mice was not sufficiently strong to depress PS generation in a baseline condition. Moreover, although VAChT$\mathrm{KD}^{\mathrm{HET}}$ mice have reduced $\mathrm{ACh}$ transmission by central cholinergic neurons (22), compensatory mechanisms, 

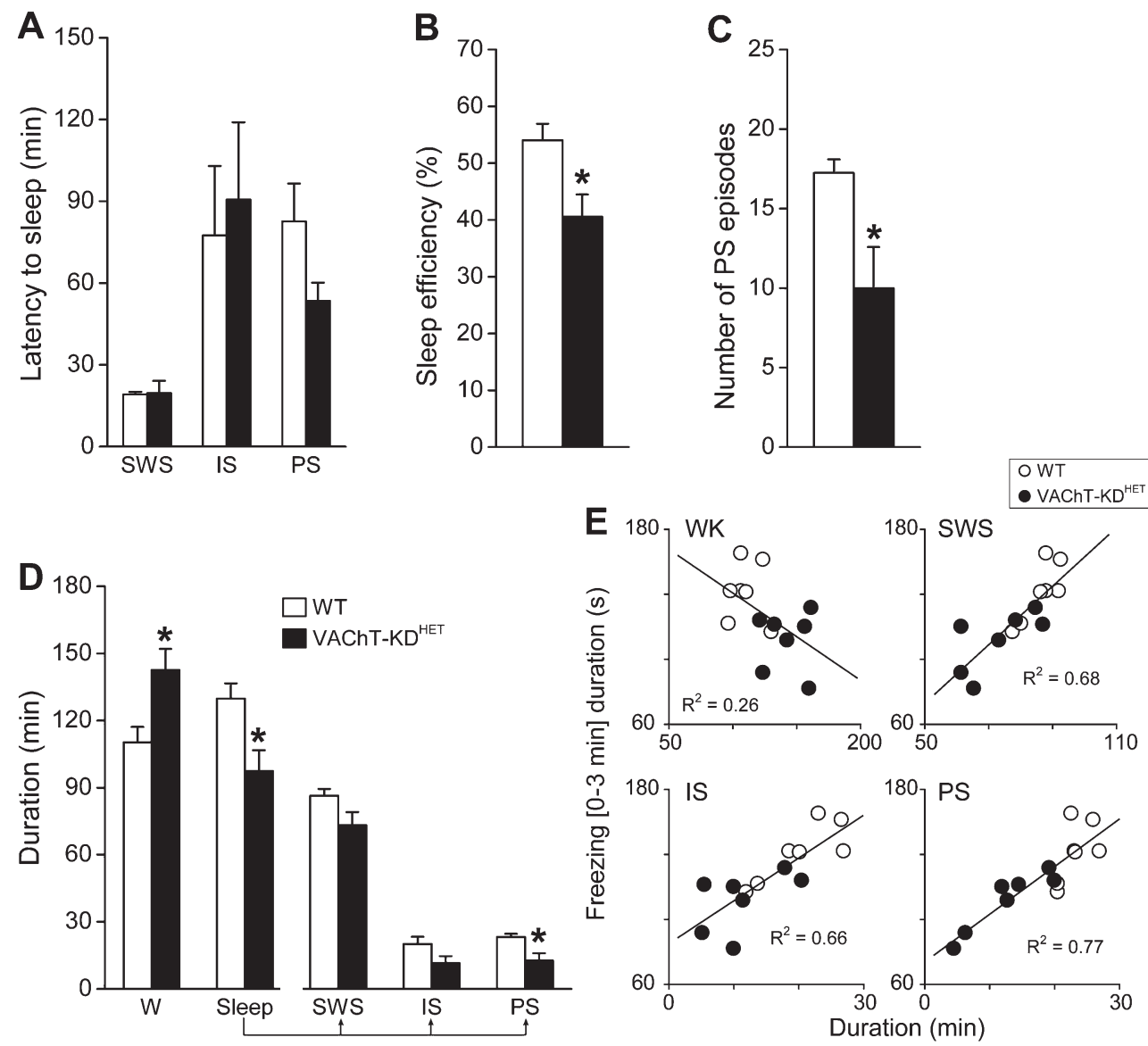

Figure 5. Impact of contextual fear conditioning test on sleep profile during the 4-h period after training. $A$, The latency to enter each of the sleep states was unaltered in VAChT-KD ${ }^{\mathrm{HET}}$ mice after training. $B$, Sleep efficiency during the 4-h period after the training session, and $C$, the number of PS episodes after the training session were significantly reduced in VAChT-KD ${ }^{\mathrm{HET}}$ animals. $D$, Averaged duration of each state during the 4-h period that followed the training session. Note that sleep reduction was mainly characterized by reduction of PS (arrows). E, Correlation between freezing duration in the first $3 \mathrm{~min}$ of the test and the duration of wake (WK), slow-wave sleep (SWS), intermediate sleep (IS), and paradoxical sleep (PS). Positive correlations (simple linear regression analysis) were observed for

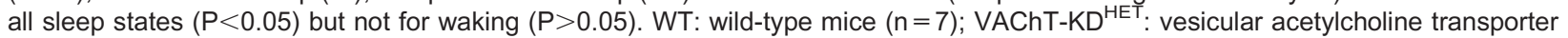
knockdown heterozygous mice $(\mathrm{n}=7)$; $\mathrm{W}$ : awake. ${ }^{*} \mathrm{P}<0.05$ (Student $t$-test).

such as supersensitivity of cholinergic receptors, could account for the normal sleep-wake physiology. Nonetheless, in a challenging situation, such as training, in which the cholinergic system is stimulated, the reduced release of ACh might have had a deeper impact in sleep physiology, leading to a reduced amount of PS (Figure 5B-D).

\section{Behavioral performance}

Our results showed impaired CFC of freezing in VAChT-KD ${ }^{\mathrm{HET}}$ mice. Previous reports showed impaired performance in cognitive tasks involving object and social recognition, but not in spatial memory or in aversive learning evaluated by the Morris water maze test and the inhibitory avoidance test, respectively $(22,23)$. Indeed, cholinergic manipulations may affect specific types of memory and may depend on different brain structures (29). Also, factors not specific to learning and memory but important for the animal's performance (i.e., attention and impulsive behavior) might have contributed to the different results. It is important to bear in mind that deficits of attention might also underlie the memory impairment observed in VAChT-KD ${ }^{\mathrm{HET}}$ mice, since nicotinic (30) and muscarinic (31) receptors have been implicated in this cognitive process. Future experiments will be necessary to reveal whether attention-dependent learning impairment occurs in VAChT-deficient mice. Other aspects, such as stress response, are discussed below.

\section{Behavioral performance: relationship to sleep induction}

The present results revealed a negative impact of 
CFC on sleep duration (but not sleep latency) following training (Figure 5) and impaired performance on the following day in VAChT-KD ${ }^{\mathrm{HET}}$ animals (testing session, Figure 5). We also found a positive correlation between PS duration after training and freezing behavior (Figure $5 E)$. Classically, PS has been involved with mnemonic processes, since its presence has been linked to the acquisition that takes place in memory-dependent tasks (32). It is known that PS deprivation before and after training impairs memory formation (33). A positive correlation has been observed between performance in the learning task and the increase in PS after training (34), and animals that did not show an increase in PS did not learn the task (35). In general, the sleep alteration observed in these studies occurs at specific time points after learning, from up to $5 \mathrm{~h}(27)$ to several days, depending on the task studied (for a review, see Ref. 32). Thus, one could speculate that sleep profile after training might account for the sleep-dependent memory impairment in VAChT-KD ${ }^{\mathrm{HET}}$ animals, albeit no causal relationship can be concluded from the experiments performed in the present study.

\section{Sleep, stress and cholinergic neurotransmission: clinical implications}

Animal models for neurodegenerative diseases that involve cholinergic neurotransmission show cognitive deficits and altered SWC similar to the human condition (21). Additionally, old (but not young) beta-2 nicotinic receptor knockout mice displayed impaired CFC freezing (36), and although no sleep recording was performed after training in these animals, one could argue that sleep alterations might underlie, or at least accompany, memory deficits. It remains to be further explored if VAChT-KD ${ }^{\mathrm{HET}}$ animals demonstrate greater sleep and memory alterations with aging. Conversely, young beta-2 nicotinic receptor knockout mice present longer PS episodes and less fragmented sleep during baseline, despite the fact that, in response to immobilization stress, mutant mice display no increase in the amount of PS and increased fragmentation compared to WT animals (19). In the present study, however, we found alterations of sleep (PS) amount after training but not during basal recordings. In both cases, it appears that stressful procedures alter sleep homeostasis in mutant animals and highlight possible differential mechanisms for nicotinic and muscarinic receptors in stress-induced sleep alterations.

While dealing with an aversive task, one could speculate whether stress reactivity or foot shock sensitivity could account for the differences observed in both sleep alteration and behavioral analysis. Indeed, sleep fragmentation and increased latency are also often reported after foot shock stress (37). In the same way,
ACh and hypothalamic-pituitary-adrenal (HPA) axis hormones are related, insofar as stressful situations stimulate $\mathrm{ACh}$ release in the septo-hippocampal cholinergic system (38) and cholinergic stimulation induces HPA axis activation, measured by ACTH and corticosterone increase (39). One could thus argue that the reduced cholinergic activity observed in VAChT-KD ${ }^{\mathrm{HET}}$ mice could lead to a stress-induced sleep alteration response, rather than a learning-induced sleep change. If this were the case, reduced ACh tonus would decrease stimulation of the HPA axis, resulting in a reduction in the amount of corticosterone released. In that case, VAChT-KD ${ }^{\mathrm{HET}}$ mice would be less affected by stress-induced sleep alterations. On the other hand, it has been shown that cholinergic deafferentation of the hippocampus results in a delay in the decline of corticosterone levels, without interfering with the peak corticosterone response after stress (40). If this were the case in VAChT-KD ${ }^{\mathrm{HET}}$ mice, one would expect a greater effect on sleep, especially on sleep latency. Our results showed no difference between VAChT-KD ${ }^{\mathrm{HET}}$ and WT mice in this parameter. Mutant mice had an even smaller PS latency (although not reaching statistical significance). Taken together, the present data suggest that different stress responses were not the main cause of sleep alterations observed in the VAChT-deficient mice.

In conclusion, VAChT-KD ${ }^{\mathrm{HET}}$ animals present similar amounts of each sleep state in comparison to WT animals while SWC were highly fragmented in the former. VAChT$\mathrm{KD}^{\mathrm{HET}}$ animals also performed poorly in a CFC paradigm, which was followed by reduced PS after the training session. To the authors' knowledge, this is the first demonstration that VAChT manipulation can alter sleep patterns with possible consequences to learning and memory processes. Our results support the notion that cholinergic neurotransmission plays an important role in sustaining PS after an enriched/stressful experience, with special significance for learning and memory. VAChT$\mathrm{KD}^{\mathrm{HET}}$ mice are thus an interesting animal model for the study of combined SWC and memory dysfunction and for their contribution to the understanding of the physiological alterations that take place in hypocholinergic states, as in Alzheimer's disease and other forms of dementia (14).

\section{Acknowledgments}

We thank Waldemarks Aires Leite for his support in surgery procedures and animal care, Dr. Vânia D'Almeida and Dr. Heloisa Allegro Baptista for assistance in genotyping animals and Dr. Gui Mi Ko and Raquel Prestes for the maintenance of the animal colony at CEDEME/UNIFESP. Research supported by AFIP, FAPESP (CEPID \#1998/14303-3) and CAPES. 


\section{References}

1. Everitt BJ, Robbins TW. Central cholinergic systems and cognition. Annu Rev Psychol 1997; 48: 649-684, doi: 10.1146/annurev.psych.48.1.649.

2. Datta S, Siwek DF. Excitation of the brain stem pedunculopontine tegmentum cholinergic cells induces wakefulness and REM sleep. J Neurophysiol 1997; 77: 2975-2988.

3. Steriade M, McCarley RW. Brainstem control of wakefulness and sleep. New York: Plenum Press; 1990.

4. Lee MG, Hassani OK, Alonso A, Jones BE. Cholinergic basal forebrain neurons burst with theta during waking and paradoxical sleep. J Neurosci 2005; 25: 4365-4369, doi: 10.1523/JNEUROSCI.0178-05.2005.

5. Jones BE. Activity, modulation and role of basal forebrain cholinergic neurons innervating the cerebral cortex. Prog Brain Res 2004; 145: 157-169, doi: 10.1016/S00796123(03)45011-5.

6. Vanderwolf $\mathrm{CH}$. Neocortical and hippocampal activation relation to behavior: effects of atropine, eserine, phenothiazines, and amphetamine. J Comp Physiol Psychol 1975; 88: 300-323, doi: $10.1037 / \mathrm{h} 0076211$.

7. Kilgard MP, Merzenich MM. Cortical map reorganization enabled by nucleus basalis activity. Science 1998; 279: 1714-1718, doi: 10.1126/science.279.5357.1714.

8. Eschenko O, Ramadan W, Molle M, Born J, Sara SJ. Sustained increase in hippocampal sharp-wave ripple activity during slow-wave sleep after learning. Learn Mem 2008; 15: 222-228, doi: 10.1101/lm.726008.

9. Stickgold R. Sleep-dependent memory consolidation. Nature 2005; 437: 1272-1278, doi: 10.1038/nature04286.

10. Bartus RT, Dean RL III, Beer B, Lippa AS. The cholinergic hypothesis of geriatric memory dysfunction. Science 1982; 217: 408-414, doi: 10.1126/science.7046051.

11. Hasselmo ME. Neuromodulation: acetylcholine and memory consolidation. Trends Cogn Sci 1999; 3: 351-359, doi: 10.1016/S1364-6613(99)01365-0.

12. Bueno OF, Oliveira GM, Lobo LL, Morais PR, Melo FH, Tufik S. Cholinergic modulation of inhibitory avoidance impairment induced by paradoxical sleep deprivation. Prog Neuropsychopharmacol Biol Psychiatry 2000; 24: 595-606, doi: 10.1016/S0278-5846(00)00095-6.

13. Reynolds CF III, Spiker DG, Hanin I, Kupfer DJ. Electroencephalographic sleep, aging, and psychopathology: new data and state of the art. Biol Psychiatry 1983; 18: 139-155.

14. McKinney M, Jacksonville MC. Brain cholinergic vulnerability: relevance to behavior and disease. Biochem Pharmacol 2005; 70: 1115-1124, doi: 10.1016/j.bcp.2005. 05.019.

15. Francis PT, Palmer AM, Snape M, Wilcock GK. The cholinergic hypothesis of Alzheimer's disease: a review of progress. J Neurol Neurosurg Psychiatry 1999; 66: 137147, doi: 10.1136/jnnp.66.2.137.

16. Okawa M, Mishima K, Hishikawa Y, Hozumi S, Hori H, Takahashi K. Circadian rhythm disorders in sleep-waking and body temperature in elderly patients with dementia and their treatment. Sleep 1991; 14: 478-485.

17. Prinz PN, Vitaliano PP, Vitiello MV, Bokan J, Raskind M, Peskind E, et al. Sleep, EEG and mental function changes in senile dementia of the Alzheimer's type. Neurobiol Aging
1982; 3: 361-370, doi: 10.1016/0197-4580(82)90024-0.

18. Bixler E. Sleep and society: an epidemiological perspective. Sleep Med 2009; 10 (Suppl 1): S3-S6, doi: 10.1016/ j.sleep.2009.07.005

19. Lena C, Popa D, Grailhe R, Escourrou P, Changeux JP, Adrien J. Beta2-containing nicotinic receptors contribute to the organization of sleep and regulate putative microarousals in mice. J Neurosci 2004; 24: 5711-5718, doi: 10.1523/JNEUROSCI.3882-03.2004.

20. Fonck C, Cohen BN, Nashmi R, Whiteaker $P$, Wagenaar DA, Rodrigues-Pinguet $\mathrm{N}$, et al. Novel seizure phenotype and sleep disruptions in knock-in mice with hypersensitive alpha $4^{*}$ nicotinic receptors. J Neurosci 2005; 25: 1139611411, doi: 10.1523/JNEUROSCI.3597-05.2005.

21. Wisor JP, Edgar DM, Yesavage J, Ryan HS, McCormick $\mathrm{CM}$, Lapustea N, et al. Sleep and circadian abnormalities in a transgenic mouse model of Alzheimer's disease: a role for cholinergic transmission. Neuroscience 2005; 131: 375-385, doi: 10.1016/j.neuroscience.2004.11.018.

22. Prado VF, Martins-Silva C, de Castro BM, Lima RF, Barros $D M$, Amaral E, et al. Mice deficient for the vesicular acetylcholine transporter are myasthenic and have deficits in object and social recognition. Neuron 2006; 51: 601-612, doi: 10.1016/j.neuron.2006.08.005.

23. de Castro BM, Pereira GS, Magalhaes V, Rossato JI, De Jaeger X, Martins-Silva C, et al. Reduced expression of the vesicular acetylcholine transporter causes learning deficits in mice. Genes Brain Behav 2009; 8: 23-35, doi: 10.1111/ j.1601-183X.2008.00439.x.

24. de Castro BM, De Jaeger X, Martins-Silva C, Lima RD, Amaral E, Menezes C, et al. The vesicular acetylcholine transporter is required for neuromuscular development and function. Mol Cell Biol 2009; 29: 5238-5250, doi: 10.1128/ MCB.00245-09.

25. Gottesmann C. The transition from slow-wave sleep to paradoxical sleep: evolving facts and concepts of the neurophysiological processes underlying the intermediate stage of sleep. Neurosci Biobehav Rev 1996; 20: 367-387, doi: 10.1016/0149-7634(95)00055-0.

26. Nail-Boucherie K, Dourmap N, Jaffard R, Costentin J. Contextual fear conditioning is associated with an increase of acetylcholine release in the hippocampus of rat. Brain Res Cogn Brain Res 2000; 9: 193-197, doi: 10.1016/S09266410(99)00058-0.

27. Graves LA, Heller EA, Pack AI, Abel T. Sleep deprivation selectively impairs memory consolidation for contextual fear conditioning. Learn Mem 2003; 10: 168-176, doi: 10.1101/ Im.48803.

28. Capece ML, Efange SM, Lydic R. Vesicular acetylcholine transport inhibitor suppresses REM sleep. Neuroreport 1997; 8: 481-484, doi: 10.1097/00001756-19970120000021.

29. Parent MB, Baxter MG. Septohippocampal acetylcholine: involved in but not necessary for learning and memory? Learn Mem 2004; 11: 9-20, doi: 10.1101/Im.69104.

30. Levin ED, McClernon FJ, Rezvani AH. Nicotinic effects on cognitive function: behavioral characterization, pharmacological specification, and anatomic localization. Psychopharmacology 2006; 184: 523-539, doi: 10.1007| 
s00213-005-0164-7.

31. Hodges DB Jr, Lindner MD, Hogan JB, Jones KM, Markus EJ. Scopolamine induced deficits in a battery of rat cognitive tests: comparisons of sensitivity and specificity. Behav Pharmacol 2009; 20: 237-251, doi: 10.1097/FBP.0b013 e32832c70f5.

32. Smith C. Sleep states, memory processes and synaptic plasticity. Behav Brain Res 1996; 78: 49-56, doi: 10.1016/ 0166-4328(95)00218-9.

33. Tiba PA, Oliveira MG, Rossi VC, Tufik S, Suchecki D. Glucocorticoids are not responsible for paradoxical sleep deprivation-induced memory impairments. Sleep 2008; 31 : 505-515.

34. Portell-Cortes I, Marti-Nicolovius M, Segura-Torres $\mathrm{P}$, Morgado-Bernal I. Correlations between paradoxical sleep and shuttle-box conditioning in rats. Behav Neurosci 1989; 103: 984-990, doi: 10.1037/0735-7044.103.5.984.

35. Smith C, Wong PT. Paradoxical sleep increases predict successful learning in a complex operant task. Behav Neurosci 1991; 105: 282-288, doi: 10.1037/0735-7044. 105.2.282.

36. Caldarone BJ, Duman $\mathrm{CH}$, Picciotto MR. Fear conditioning and latent inhibition in mice lacking the high affinity subclass of nicotinic acetylcholine receptors in the brain. Neuropharmacology 2000; 39: 2779-2784, doi: 10.1016/ S0028-3908(00)00137-4.

37. Vazquez-Palacios G, Velazquez-Moctezuma J. Effect of electric foot shocks, immobilization, and corticosterone administration on the sleep-wake pattern in the rat. Physiol Behav 2000; 71: 23-28, doi: 10.1016/S0031-9384(00) 00285-7.

38. Gilad GM. The stress-induced response of the septohippocampal cholinergic system. A vectorial outcome of psychoneuroendocrinological interactions. Psychoneuroendocrinology 1987; 12: 167-184, doi: 10.1016/0306-4530(87) 90002-3.

39. Bugajski AJ, Gadek-Michalska A, Bugajski J. The involvement of nitric oxide and prostaglandins in the cholinergic stimulation of hypothalamic-pituitary-adrenal response during crowding stress. J Physiol Pharmacol 2006; 57: 463477.

40. Han JS, Bizon JL, Chun HJ, Maus CE, Gallagher M. Decreased glucocorticoid receptor mRNA and dysfunction of HPA axis in rats after removal of the cholinergic innervation to hippocampus. Eur J Neurosci 2002; 16: 1399-1404, doi: 10.1046/j.1460-9568.2002.02191.x. 